\title{
Urethral Diverticulum: Unusual Cause of Urinary Incontinence after a Radical Prostatectomy
}

\author{
Nabil Jakhlal ${ }^{1,2}$, Amine Slaoui ${ }^{1,2}$, Alae Touzani ${ }^{1,2}$, Tariq Karmouni ${ }^{1,2}$, Khalid El Khader ${ }^{1,2}$, Abdellatif Koutani ${ }^{1,2}$, \\ Ahmed Iben Attya Andaloussi ${ }^{1,2}$ \\ ${ }^{1}$ Faculty of Medecine and Pharmacy, Mohammed V University, Rabat, Morocco \\ ${ }^{2}$ Departement of Urology, Ibn Sina Teaching Hospital, Rabat, Morocco
}

Submission: July 21, 2017; Published: September 25, 2017

*Corresponding author: Nabil Jakhlal, Department of Urology B, Mohammed V University, Morocco, Email: doc.nabil54@gmail.com

\begin{abstract}
A 63-year-old hypertensive patient presented urinary incontinence following radical prostatectomy. Urethro-cystoscopy revealed an urethral diverticulum. The cure of the latter allowed a complete disappearance of the incontinence. The purpose of this work is to first describe the characteristics of male urethral diverticula and, above all, to recall the extreme importance of morphological exploration of the lower urinary tract before considering any surgical treatment of urinary incontinence post-radical prostatectomy.
\end{abstract}

Keywords: Urethral diverticulum; Urinary incontinence; Radical prostatectomy

Abbreviations: UD: Urethral diverticulum

\section{Introduction}

Urethral diverticulum (UD) is defined as a tubular or sacular dilatation that opens into the urethra [1]. It is rare in men and can be congenital or acquired [2]. Its clinical symptomatology is non-specific. Urethro-cystoscopy or urethro-cystography retrograde and voiding make it possible to establish its diagnosis. We present a case of a DU manifested by urinary incontinence in a patient with history of radical prostatectomy.

\section{Observation}

A 63-year-old patient with no specific pathological history except for well-controlled hypertension under a single anti hypertensive, consults for a PSA of 7.89ng/ml. Moreover, it does not show any urinary signs of the lower-device and the rectal examination finds a prostate gland of $50 \mathrm{~g}$ and supple. Prostate biopsy revealed prostate adenocarcinoma, Gleason $6(3+3)$. Three out of 13 carrots are positive, located at the base and middle left, measuring $11 / 137 \mathrm{~mm}$. There is no peri-nervous invasion or vascular invasion. Peri-capsular tissue is not shown. Multi parametric prostatic magnetic resonance imagine grevealed a suspect nodule at the level of the left peripheral area, measuring $17 * 12 \mathrm{~mm}$.

The patient underwent a radical prostatectomy without preservation of the vasculo-nerve strips with bilateral pelvic dissection. The anatomopathological analysis of the surgical specimen confirmed the diagnosis of prostate adenocarcinoma,
Gleason $6(3+3)$, infiltrating the 2 prostatic lobes with intraprostatic perinervous involvement without vascular emboli. Surgical margins were focally affected at the lower right lobar level and at the peri-cervical prostatic parenchyma. The lymph node dissection was negative.

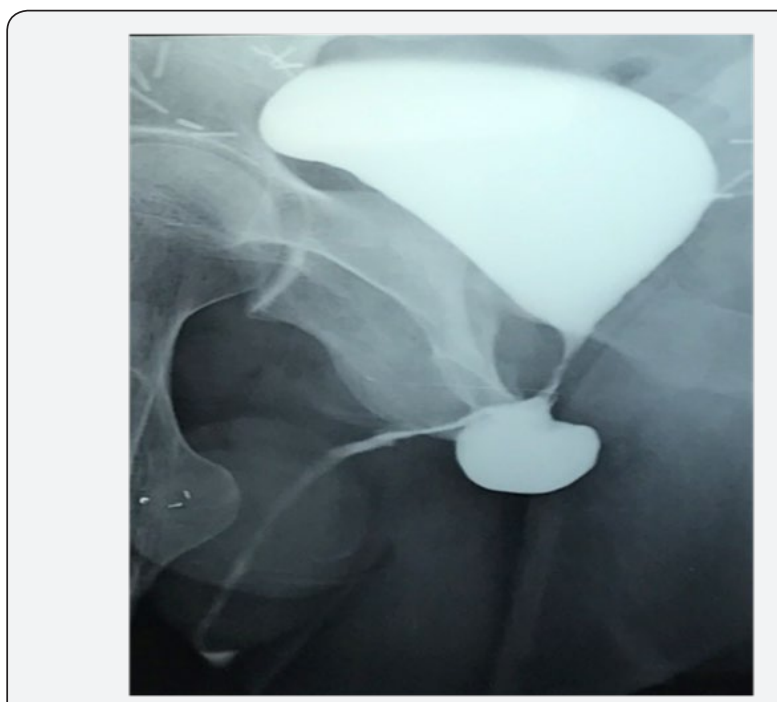

Figure 1: Urethro-cystography showing a urethral divercule at the bulbar level.

After discussion with the patient, we opted for surveillance without performing adjuvant radiotherapy. The control PSA was 


\section{Global Journal of Reproductive Medicine}

undetectable with a one-year follow-up. Nevertheless, the patient still complained of permanent urinary leaks with preservation of urination. Urethro-cystoscopy has demonstrated an urethral diverticulum located at the level of the bulbar urethra. Urethro- cystography retrograde and voiding confirms this diagnosis Figure 1. After removal of the diverticulum and suture of the urethra Figure 2, the progression was favorable with a follow-up of 6 months.

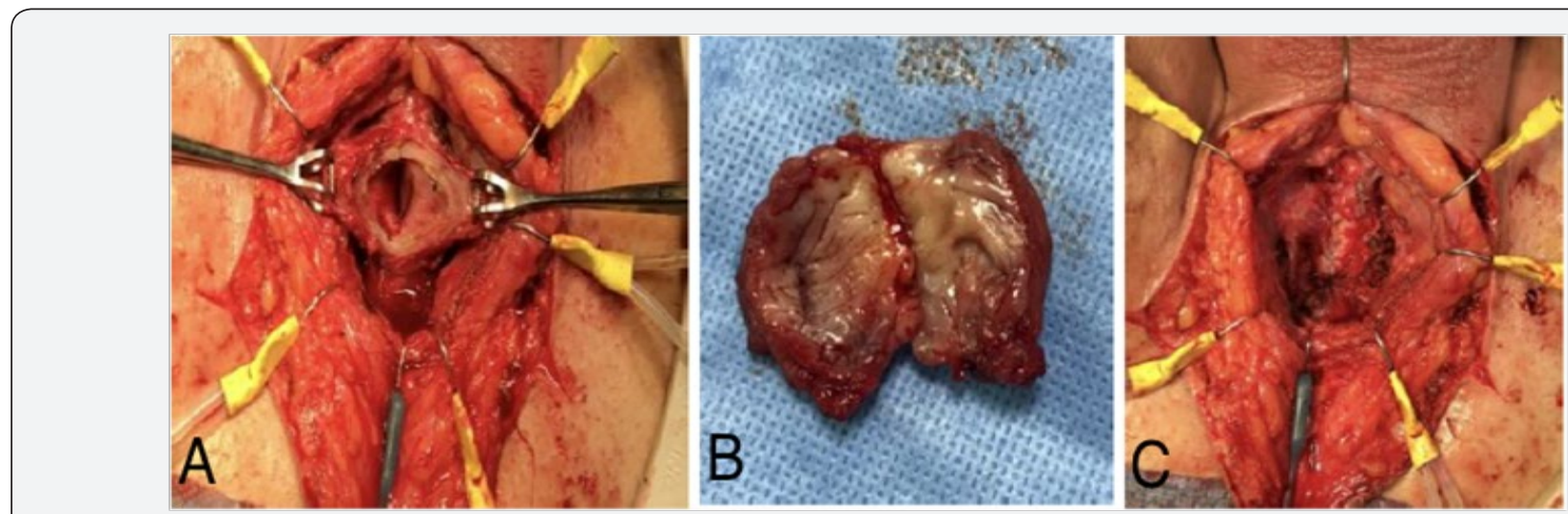

Figure 2: Peroperative images of the opening of the diverticulum (A), its excision (B) and the suture of the urethra (C).

\section{Discussion}

The urethral diverticulum (UD) is defined as a tubular or sacular dilatation that opens into the urethra through an ostium at any point along its path [1]. Congenital or acquired, primary or secondary it is rare in men in effect, only a few cases have been reported in the literature [2]. Congenital is less than $10 \%$ of cases and it is the apange of children with urinary obstruction [2]. Many hypotheses have been proposed to explain the development of congenital UD. Among the many hypotheses proposed to explain its development, the partial lack of the spongy tissue leading to a defective closure of the bulbous part of the urethra defended by Voillemier L.D in 1868 seems to be the most valid [3,4]. It is usually located in the ventral part of the anterior urethra.

While more than $90 \%$ of UD cases in men are acquired. A multitude of etiopathogenic theories have been published in the literature:

o Increased urethral pressure upstream of an obstruction that causes hernia of the urethral epithelium, observed in patients with a history of reconstructions of hypospadias, urethral stricture or trauma [5].

o The constant pressure on the penoscrotal angle could induce urethral ischemia then fibrosis and finally the formation of scars. This can be seen in men with indwelling catheters [5].

o Ganabathi et al. [6] suggested that rupture of the periurethral glands in the urethral lumen secondary to obstruction could lead to epithelization and the formation of a periurethral cavity. An additional stress causes dilation and an increase in the size of the diverticulum [7].

o The acquired UD may be due to suppuration and necrosis of the urethral wall following trauma, endourethral manipulation or drainage of a prostatic or periurethral abscess [8]. o In our case, given the absence of a sign previous to radical prostatectomy, the congenital origin seems eliminated at the outset. Thus, it is likely to be secondary to endo-urethral manipulations during radical prostatectomy and or postoperative bladder catheterization.

UD may be asymptomatic, however patients often report nonspecific lower urinary tract symptoms: recurrent urinary tract infection, pelvic pain, incontinence, post-voiding, dysuria, pollakiuria, urgenturia, nocturia or even a feeling of incomplete emptying of the bladder. On the other hand, it can also be manifested by a perineal mass [8,9]. It is important to remember that urinary tract infection, fistulas and computational formation in the diverticulum are the most common complications [7].

Radiological diagnosis is essential to plan its therapeutic management. Indeed urethro-cystoscopy often makes it possible to discover the DU. Urethrocystography retrograde and voiding in association with ultrasound are the best examinations to confirm the diagnosis and characterize the DU [5,9]. In addition to specifying the location and size of the diverticulum, it informs the thickness of the diverticulum wall about the size of the collar and also provides information about the proximal and distal urethra to the diverticulum [8].

The management of urethral diverticula is not codified because of the rarity of this pathology; It is suggested that a small asymptomatic diverticulum would not require intervention. However, the patient must imperatively manually compress the diverticulum after each urination in order to avoid urinary stasis and the occurrence of other complications $[1,2]$.

Endoscopic treatment offers a relatively a traumatic and simple solution. However, it is necessary to eliminate first of all certain situations which could lead to a bad evolution:

o A surrounding supporting tissue deficient,

o A large, poorly drained cavity likely to be left behind 


\section{Global Journal of Reproductive Medicine}

o A diverticulum is too thick or fibrous to be adequately incised [3].

In addition, many authors have hypothesized that endoscopic treatment increases the risk of urethro-cutaneous fistula [10]. Thus in patients previously selected well Allen and all obtained very good results [11]. In cases where endoscopic treatment is not appropriate, open surgery should be used. The diverticulum should be excised completely, the urethra should be restored and, if necessary, additional tissue should be provided to enhance repair and thus prevent skin fistula. The simplest surgical option to achieve these goals should be preferred. Excision and primary anastomosis are technically the least demanding. However, care must be taken to ensure that the repair has a good blood supply, is not energized, and uses good quality tissue without infection [11]. A Urinary diversion may then be proposed in patients with other associated abnormalities of the lower urinary tract such as a neurogenic bladder or artificial urinary sphincter [5].

\section{Conclusion}

Our patient presented permanent urinary incontinence with preservation of urination after radical prostatectomy. Given the context, this urinary incontinence could be considered as a complication of radical prostatectomy, the mechanisms of which have nothing to do with UD. The fortuitous discovery of the latter with urethro-cystoscopy changed the whole therapeutic management. Thus, this work makes it possible to recall the indispensable character of the morphological exploration of the lower urinary tract before considering any surgical treatment of urinary incontinence post-radical prostatectomy.

\section{References}

1. Mohan V, Gupta SK, Cherian J, Tripathi VN, Sharma BB (1980) Urethral diverticulum in male subjects report of 5 cases. J Urol 123(4): 592-594.

2. Monish A, Rajeev TP, Dorairajan LN, Gupta NP (1999) Infected congenital urethral diverticulum in an adult male. UrolInt 62(3): 177179.

3. Ortlip SA, Gonzalez R, Williams RD (1980) Diverticula of the male urethra. J Urol 124(3): 350-355.

4. Sen SE, Iseri C, Eryigit M (1989) Congenital urethral diverticulum in the male. Urology 34: 129-30.

5. Cinman NM, McAninch JW, Glass AS, Zaid UB, Breyer BN (2012) Acquired male urethral diverticula Presentation, diagnosis and management. J Urol 188(4): 1204-1208.

6. Ganabathi K, Leach GE, Zimmern PE, Dmochowski R (1994) Experience with the management of urethral diverticulum in 63 women. J Urol 152 (5 Pt 1): 1445-1452.

7. Popoola AA, Oyinloye OI, Aremu IB (2009) Acquired male urethral diverticulum Case reports. Niger Postgrad Med J 16(3): 224226.

8. Mohanty D, Garg P, Jain B, Bhatt S (2014) Male urethral diverticulum having multiple stones. Ann Med Health Sci Res 4(1): S53S55.

9. El Ammari JE, Riyach O, Ahsaini M, Ahallal Y, El Fassi MJ, et al. (2012) Acquired urethral diverticulum in a man with paraplegia presenting with ascrotal mass a case report. J Med Case Rep 6: 392.

10. Jones EA, Freedman AL, Ehrlich RM (2002) Megalourethra and urethral diverticula. UrolClin North Am 29(2): 341-348.

11. Allen D, Mishra V, Pepper W, Shah S, Motiwala H (2007) A Single-Center Experience of Symptomatic Male Urethral Diverticula. Urology 70(4): 650-653.

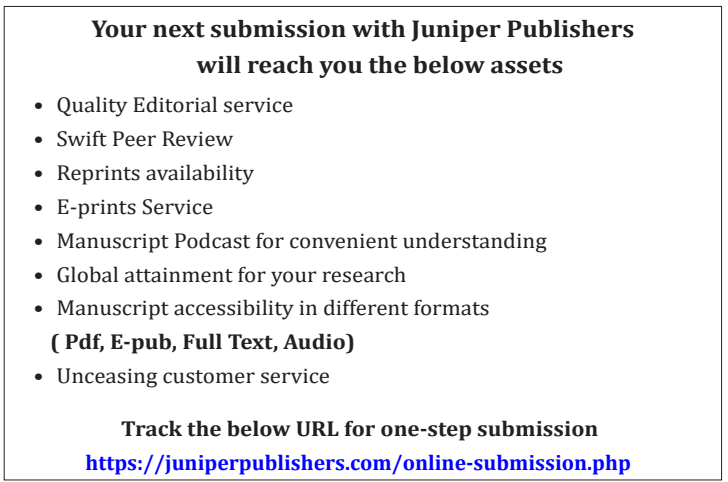

